\title{
Translation and adaptation of the Bipolar Prodrome Symptom Scale-Retrospective - Patient Version to Brazilian Portuguese
}

\author{
Tradução e adaptação da Bipolar Prodrome Symptom Scale-Retrospective - \\ Patient Version para português brasileiro
}

Pedro Mario Pan, ${ }^{1}$ Danilo Rocha de Jesus, ${ }^{2}$ Ary Gadelha, ${ }^{1}$ Rodrigo Affonseca Bressan, ${ }^{1}$ Christoph U. Correll, ${ }^{3}$ Rodrigo Barbachan Mansur, ${ }^{1}$ André Zugman, ${ }^{1}$ Cristiano Noto, ${ }^{1}$ Elson de Miranda Asevedo, ${ }^{1}$ Elisa Brietzke ${ }^{1}$

\begin{abstract}
Background: Bipolar disorder (BD) is a chronic and often severe mental disease, associated with a significant burden in affected individuals. The characterization of a premorbid (prodromal) period and possible development of preventive interventions are recent advances in this field. Attempts to characterize high-risk stages in $B D$, identifying symptoms prior to the emergence of a first manic/hypomanic episode, have been limited by a lack of standardized criteria and instruments for assessment. The Bipolar Prodrome Symptom Scale-Retrospective (BPSS-R), developed by Correll and collaborators, retrospectively evaluates symptoms that occur prior to a first full mood episode in individuals with $B D$.

Objective: To describe the translation and adaptation process of the BPSS-R to Brazilian Portuguese.

Method: Translation was conducted as follows: 1) translation of the scale from English to Brazilian Portuguese by authors who have Portuguese as their first language; 2 ) merging of the two versions by a committee of specialists; 3 ) back-translation to English by a translator who is an English native speaker; 4) correction of the new version in English by the author of the original scale; 5) finalization of the new version in Brazilian Portuguese. Results: All the steps of the translation process were successfully accomplished, resulting in a final version of the instrument. Conclusions: The Brazilian Portuguese version of the BPSS-R is a potentially useful instrument to investigate prodromal period of $\mathrm{BD}$ in Brazil.
\end{abstract}

Keywords: Bipolar disorder, at-risk mental sates, questionnaire, prodromal symptoms.

\section{Resumo}

Introdução: $O$ transtorno bipolar (TB) é um transtorno mental crônico e muitas vezes grave, associado a um significativo prejuízo psicossocial nos indivíduos afetados. A caracterização de um período pré-mórbido (prodrômico) e o possível desenvolvimento d e intervenções preventivas são avanços recentes na área. Tentativas de caracterizar estágios de alto risco para o TB, através da identificação de sintomas antes do aparecimento de um primeiro episódio maníaco/hipomaníaco, têm sido limitadas pela falta de critérios padronizados e instrumentos de avaliação. A Bipolar Prodrome Symptom Scale-Retrospective (BPSS-R), desenvolvida por Correll e colaboradores, avalia retrospectivamente os sintomas que ocorrem antes de um episódio sindrômico de humor em indivíduos com TB. Objetivo: Descrever o processo de tradução e adaptação da BPSS-R para português brasileiro.

Método: A tradução foi conduzida como segue: 1) tradução da escala de inglês para português brasileiro por autores que têm o português como língua materna; 2) junção das duas versões por um comitê de especialistas; 3) retrotradução para inglês por um tradutor que tem inglês como língua materna; 4) correção da nova versão em inglês pelo autor do instrumento original; 5) finalização da nova versão em português brasileiro.

Resultados: Todos os passos do processo de tradução foram completados com sucesso, resultando em uma versão final do instrumento.

Conclusões: A versão da BPSS-R em português brasileiro é um instrumento potencialmente útil para investigar o período prodrômico do TB no Brasil.

Descritores: Transtorno bipolar, estados mentais de risco, questionário, sintomas prodrômicos.

\footnotetext{
${ }_{1}^{1}$ Program for Recognition and Intervention in Individuals in At-Risk Mental States (PRISMA), Department of Psychiatry, Universidade Federal de São Paulo (UNIFESP), São Paulo, SP, Brazil. Interdisciplinary Laboratory of Clinical Neurosciences (LINC), UNIFESP. ${ }^{2}$ Centre for Addiction and Mental Health, University of Toronto, Toronto, Ontario, Canada. ${ }^{3}$ The Zucker Hillside Hospital, Psychiatry Research, North Shore - Long Island Jewish Health System, Glen Oaks, NY, USA. Albert Einstein College of Medicine, Bronx, NY, USA. The Feinstein Institute for Medical Research, Manhasset, NY, USA. Hofstra North Shore LIJ School of Medicine, Hempstead, NY, USA. Financial support: none.

Submitted Feb 06 2012, accepted for publication Apr 05 2012. No conflicts of interest declared concerning the publication of this article.

Suggested citation: Pan PM, de Jesus DR, Gadelha A, Bressan RA, Correll CU, Mansur RB, et al. Translation and adaptation of the Bipolar Prodrome Symptom ScaleRetrospective - Patient Version to Brazilian Portuguese. Trends Psychiatry Psychother. 2013;35(1):62-75.
} 


\section{Introduction}

Bipolar disorder (BD) is a chronic, highly prevalent, potentially severe mood disorder, associated with significant suffering, functional impairment, and higher mortality rates compared with the general population. ${ }^{1,2}$ In addition, $\mathrm{BD}$ requires long-term pharmacological and psychosocial treatment, in most cases with only partial efficacy. ${ }^{3}$ Therefore, strategies that can help prevent BD, reduce symptom severity and functional deficits, and possibly delay its onset are needed.

Lack of knowledge of the psychopathology and neurobiology of the transition to the first mood episode limits research in the primary prevention of BD. ${ }^{4}$ Consequently, delayed and often mistaken diagnoses are made, leading to exposure of a large number of individuals with BD to antidepressants, benzodiazepines, and psychostimulants, which can have potential negative effects on their symptomatology. ${ }^{4}$

Clinicians and even patients with BD largely recognize the existence of a period before the onset of the first manic/hypomanic episode in which subsyndromic symptoms are present (prodromal period). 5,6 The adequate characterization of this period has received relatively little attention until recently, but studies on prodromal psychosis have revealed promising results. 7,8 The growing evidence showing that the prodromal period is part of the evolution of $\mathrm{BD}$ resulted in the inclusion of this pre-clinical or prodromal period in BD staging models. ${ }^{9,10}$ Akiskal et al. prospectively studied 68 adolescents who had a firstdegree relative diagnosed with $\mathrm{BD}$ and were the first to suggest the existence of a prodromal stage of BD. They observed a high rate of mild mood symptoms, especially with a depressive polarity. ${ }^{11}$ After that first study, several others have tried to characterize this critical period. ${ }^{12,13}$ Because pre-clinical symptoms are not observed in all individuals with $\mathrm{BD}$, prospective studies have suggested the denomination "bipolar at-risk" or "ultra-high risk" rather than "prodromal" to refer to these patients.

One important limitation of research on the early stages of $\mathrm{BD}$ is the scarcity of instruments available to evaluate prodromal symptoms. In this scenario, the Bipolar Prodrome Symptom Scale-Retrospective (BPSS-R), developed by Correll et al., retrospectively evaluates symptoms occurring prior to the first mood episode in individuals with BD. ${ }^{14}$ The BPSS-R consists of a semi-structured interview based on a downward extension of criteria listed in the Diagnostic and Statistical Manual of Mental Disorders, 4th edition (DSM-IV) and available rating scales for BD and major depressive disorder. Development of the BPSS-R was informed by a review of the literature regarding risk factors and early symptoms of BD, previously published scales and interviews designed for the assessment of psychotic prodrome and character traits, and input from experts in the prodromal stage of schizophrenia and BD. The BPSS-R assesses the onset pattern, duration, severity, and frequency of 36 symptoms and signs that emerge or worsen prior to the first major depressive and/or first manic episode. Severity of prodromal symptoms is rated using an ordinal scale, as follows: 0 =absent, $1=$ mild, 2 = moderate, and $3=$ severe. Symptom frequency is rated as follows: 0 = absent, 1 = infrequent, 2 = moderately frequent, 3 = very frequent, and $4=$ static lifetime. ${ }^{14}$

The objective of this study was to describe the translation and adaptation of the BPSS-R to Brazilian Portuguese, in order to use the instrument in future studies designed to investigate the prodromal period of BD in Brazil.

\section{Methods}

Prior to the beginning of the translation process, consent was obtained from the author of the original scale, Dr. Christoph U. Correll. Thereafter, translation and adaptation of the BPSS-R from English to Brazilian Portuguese was performed following the guidelines proposed by Guillemin et al for the adaptation of instruments. ${ }^{15}$

The translation process included the following steps: 1) translation from English to Brazilian Portuguese by two authors who have Portuguese as their first language, one of them being a psychiatrist; 2) merging of the two versions by a committee of specialists; 3) backtranslation to English by a certified translator who is an English native speaker; 4) correction of the English version by the author of the original scale, Dr. Christoph Correll; and 5) final modifications suggested by the latter author and finalization of the new version in Portuguese. These steps are illustrated in Figure 1.

\section{Results}

All the steps of the translation process were successfully accomplished, and a final version of the instrument in Brazilian Portuguese was produced. Adaptations consisted primarily of changing words whose literal translation was not very common in Brazil. Following the methodological steps described above, only very few, minor modifications were made in comparison with the original version. Examples of excerpts that generated doubts throughout the process, as taken from the original version, their translation to Portuguese, back-translation, comments by the author of the original scale, and the final version in Portuguese are shown in Table 1 . The full version of the scale in Brazilian Portuguese can be found in Appendix 1. 


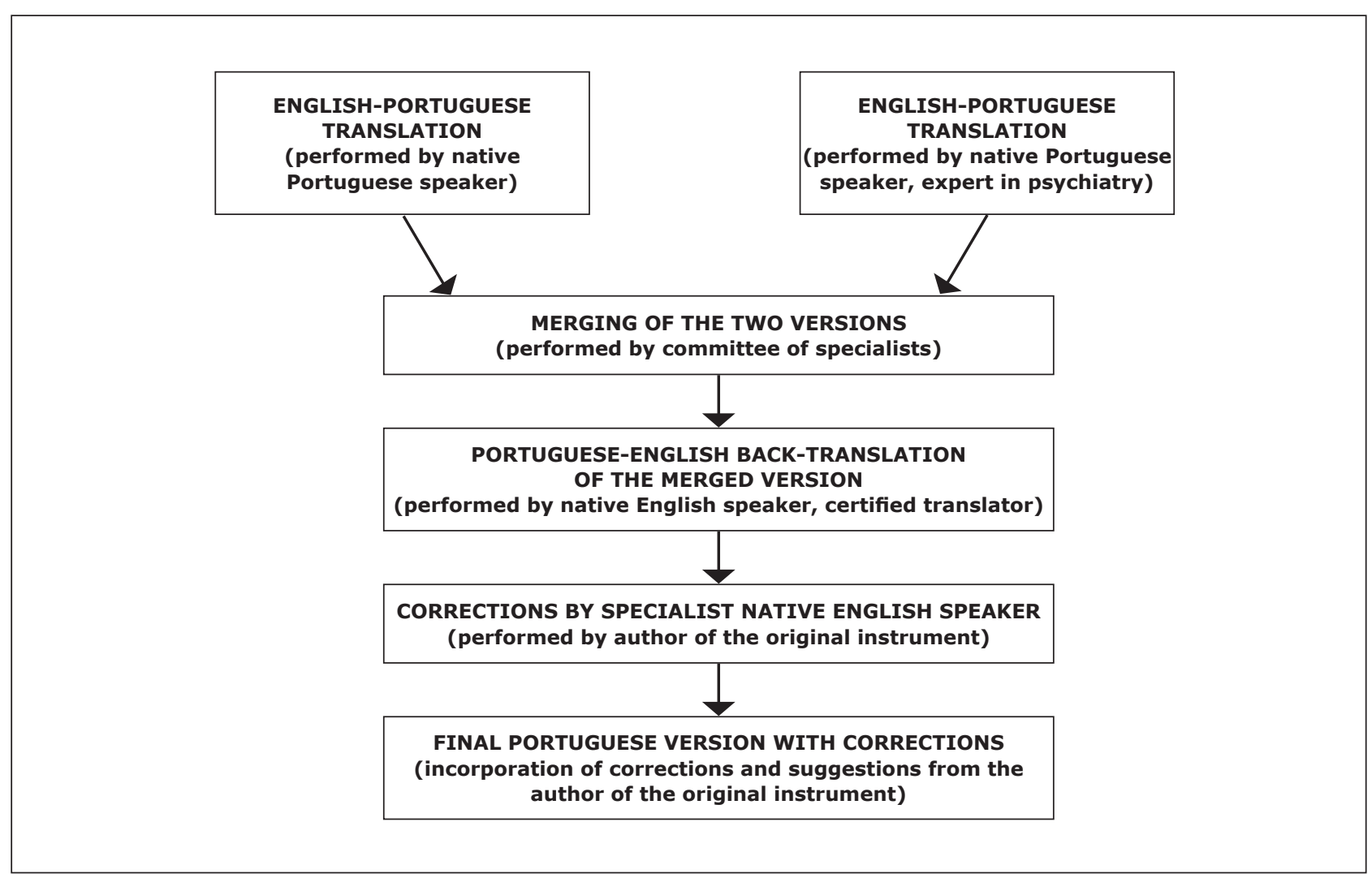

Figure 1 - Methodological steps in the translation of the Bipolar Prodrome Symptom Scale-Retrospective (BPSS-R)

Table 1 - Original version, translation to Portuguese, back-translation to English and final version in Portuguese of doubtful terms of BPSS

\begin{tabular}{|c|c|c|c|c|}
\hline Original & Translation & Back-translation & Author's comments & Final version \\
\hline $\begin{array}{l}\text { * Interviewer: note } \\
\text { timing, severity, } \\
\text { and frequency for } \\
\text { all symptoms first }\end{array}$ & $\begin{array}{l}\text { * Entrevistador: anote } \\
\text { primeiro o período, } \\
\text { gravidade e frequência } \\
\text { de todos os sintomas. }\end{array}$ & $\begin{array}{l}\text { * Interviewer: first } \\
\text { inform the period, } \\
\text { severity and frequency } \\
\text { of all symptoms. }\end{array}$ & $\begin{array}{l}\text { Instead of "inform," this should be } \\
\text { "note" or "write down." Instead of } \\
\text { "period," this should read as "timing," } \\
\text { as in time/date of the onset. }\end{array}$ & $\begin{array}{l}\text { * Entrevistador: anote } \\
\text { primeiro a época (momento/ } \\
\text { data), gravidade e frequência } \\
\text { de todos os sintomas. }\end{array}$ \\
\hline $\begin{array}{l}\text { 33. Giddy, } \\
\text { clownish }\end{array}$ & 33. Jocoso, rude & 33. Playful, rude & $\begin{array}{l}\text { "Giddy" should mean laughing for } \\
\text { no reason or laughing too much, } \\
\text { and "clownish" playing the clown, } \\
\text { making lots of jokes. }\end{array}$ & $\begin{array}{l}\text { Rindo sem motivo, "bancando } \\
\text { o palhaço". }\end{array}$ \\
\hline $\begin{array}{l}35 . \\
\text { Suspiciousness/ } \\
\text { persecutory ideas }\end{array}$ & $\begin{array}{l}\text { Desconfianças/ideias } \\
\text { persecutórias }\end{array}$ & $\begin{array}{l}\text { 35. Suspicion/ } \\
\text { persecutory ideas }\end{array}$ & $\begin{array}{l}\text { Is suspicion and suspiciousness } \\
\text { (which it should be) the same in } \\
\text { Portuguese, i.e., a thought that } \\
\text { involves fear of some form of harm } \\
\text { by others. }\end{array}$ & OK \\
\hline $\begin{array}{l}\text { Strange or unusual } \\
\text { ideas }\end{array}$ & $\begin{array}{l}\text { Ideias estranhas ou } \\
\text { incomuns }\end{array}$ & $\begin{array}{l}\text { Strange or uncommon } \\
\text { ideas }\end{array}$ & $\begin{array}{l}\text { Is "uncommon" the same as } \\
\text { "unusual," which does not refer to } \\
\text { "rare thoughts," but thoughts people } \\
\text { would not ordinarily have, i.e., more } \\
\text { bizarre or esoteric thoughts }\end{array}$ & $\begin{array}{l}\text { Ideias estranhas ou não } \\
\text { usuais }\end{array}$ \\
\hline $\begin{array}{l}\text { Difficulty making } \\
\text { decisions }\end{array}$ & $\begin{array}{l}\text { Dificuldade para tomar } \\
\text { decisões }\end{array}$ & $\begin{array}{l}\text { Difficulty taking } \\
\text { decisions }\end{array}$ & $\begin{array}{l}\text { Taking and making decisions is likely } \\
\text { synonym. }\end{array}$ & OK \\
\hline $\begin{array}{l}\text { 1. How many } \\
\text { hospitalizations } \\
\text { have you had for } \\
\text { suicidal thoughts/ } \\
\text { gestures? }\end{array}$ & $\begin{array}{l}\text { 1. Quantas } \\
\text { hospitalizações } \\
\text { você teve devido a } \\
\text { pensamentos/atitudes } \\
\text { suicidas? }\end{array}$ & $\begin{array}{l}\text { 1. How many times } \\
\text { were you admitted due } \\
\text { to suicidal thoughts/ } \\
\text { attitudes? }\end{array}$ & $\begin{array}{l}\text { This should be "gestures," which } \\
\text { means that someone insinuates } \\
\text { or acts out suicidal behavior, like } \\
\text { pointing a knife, threatening to put a } \\
\text { rope around the neck, etc. }\end{array}$ & $\begin{array}{l}\text { 1. Quantas hospitalizações } \\
\text { você teve devido } \\
\text { a pensamentos/ } \\
\text { comportamentos suicidas? }\end{array}$ \\
\hline
\end{tabular}




\section{Discussion}

The process of translation and adaptation here described was conducted by the authors, resulting in a Brazilian Portuguese version of a comprehensive instrument that retrospectively evaluates prodromal symptoms in individuals with BD.

The study of prodromal stages of severe mental disorders such as BD and schizophrenia has been a topic of great interest in psychiatry. ${ }^{7}$ The identification of such cases could offer a unique and valuable opportunity in which preventive strategies could be undertaken. 5,6,13 Although BD has been recognized as a disorder where a prodromal period is frequently reported, there is a scarcity of instruments that one can use to measure the severity, frequency, and duration of symptoms preceding the first manic/hypomanic episode. This has led to the use of non-specific scales, developed for different purposes. ${ }^{16,17}$ However, systematic studies focusing on the bipolar prodrome and using specific instruments could potentially contribute to better define this period from a psychopathological point of view. Although the prospective evaluation of individuals in at-risk mental states is seen as a more robust research design to evaluate the transition to $B D$, retrospective studies are also necessary to refine the criteria to be included in prospective instruments. ${ }^{14}$ In fact, the retrospective assessment of the prodromal stage before full schizophrenia sparked the development of prospective instruments and further research on at-risk states for schizophrenia. ${ }^{18}$

Brazil has been playing a prominent role in BD research, but the lack of translated and adapted instruments has limited advances in the field. The BPSS-R (Patient Version) is the only instrument now available in Brazilian Portuguese to evaluate the bipolar prodrome. Although the availability of this instrument is a major step forward, validation in a population with $B D$ is a necessary next step before the BPSS-R can be adopted in a number of different settings.

\section{References}

1. Fajutrao L, Locklear J, Priaulx J, Heyes A. A systematic review of the evidence of the burden of bipolar disorder in Europe. Clin Pract Epidemiol Ment Health. 2009;5:3.

2. Hoang U, Stewart R, Goldacre MJ. Mortality after hospital discharge for people with schizophrenia or bipolar disorder: retrospective study of linked English hospital episode statistics, 1999-2006. Br Med J. 2011;343:d5422.

3. Nierenberg AA, Ostacher MJ, Calabrese JR, Ketter TA, Marangell LB, Miklowitz DJ, et al. Treatment-resistant bipolar depression: a STEP-BD equipoise randomized effectiveness trial of antidepressant augmentation with lamotrigine, inositol, or risperidone. Am J Psychiatry. 2006;163:210-6.
4. Taylor M, Bressan RA, Pan Neto P, Brietzke E. Early intervention for bipolar disorder: current imperatives, future directions. Rev Bras Psiquiatr. 2011;33 Suppl 2:s197-212.

5. Correll CU, Penzner JB, Lencz T, Auther A, Smith CW, Malhotra $A K$, et al. Early identification and high-risk strategies for bipolar disorder. Bipolar Disord. 2007;9:324-38.

6. Howes OD, Falkenberg I. Early detection and intervention in bipolar affective disorder: targeting the development of the disorder. Curr Psychiatry Rep. 2011;13:493-9.

7. Correll CU, Hauser M, Auther AM, Cornblatt BA. Research in people with psychosis risk syndrome: a review of the current evidence and future directions. J Child Psychol Psychiatry. 2010;51:390-431.

8. Yung AR, Nelson B. Young people at ultra high risk for psychosis: a research update. Early Interv Psychiatry. 2011;5 Suppl 1:52-7.

9. Kapczinski F, Dias VV, Kauer-Sant'anna M, Frey BN, Grassi-Oliveira R, Colom F, et al. Clinical implications of a staging model for bipolar disorders. Expert Rev Neurother. 2009;9:957-66.

10. Berk M. Neuroprogression: pathways to progressive brain changes in bipolar disorder. Int $\mathrm{J}$ Neuropsychopharmacol. 2009;12:441-5.

11. Akiskal HS, Downs J, Jordan P, Watson S, Daugherty D, Pruitt DB. Affective disorders in referred children and younger siblings of manic-depressives. Mode of onset and prospective course. Arch Gen Psychiatry. 1985;42:996-1003.

12. Bechdolf A, Nelson B, Cotton SM, Chanen A, Thompson A, Kettle J, et al. A preliminary evaluation of the validity of atrisk criteria for bipolar disorders in help-seeking adolescents and young adults. J Affect Disord. 2010;127:316-20.

13. Leopold K, Ritter P, Correll CU, Marx C, Ozgurdal S, Juckel G, et al. Risk constellations prior to the development of bipolar disorders: rationale of a new risk assessment tool. J Affect Disord. 2012;136:1000-10.

14. Correll CU, Penzner JB, Frederickson AM, Richter JJ, Auther AM, Smith CW, et al. Differentiation in the preonset phases of schizophrenia and mood disorders: evidence in support of a bipolar mania prodrome. Schizophr Bull. 2007;33:703-14.

15. Guillemin F, Bombardier C, Beaton D. Cross-cultural adaptation of health-related quality of life measures: literature review and proposed guidelines. J Clin Epidemiol. 1993;46:1417-32.

16. Youngstrom EA, Frazier TW, Demeter C, Calabrese JR, Findling RL. Developing a 10-item mania scale from the Parent General Behavior Inventory for children and adolescents. J Clin Psychiatry. 2008;69:831-9.

17. Horwitz SM, Demeter CA, Pagano ME, Youngstrom EA, Fristad MA, Arnold LE, et al. Longitudinal Assessment of Manic Symptoms (LAMS) study: background, design, and initial screening results. J Clin Psychiatry. 2010;71:1511-7.

18. Hafner H, Riecher-Rossler A, Maurer K, Fatkenheuer B, Loffler W. First onset and early symptomatology of schizophrenia. A chapter of epidemiological and neurobiological research into age and sex differences. Eur Arch Psychiatry Clin Neurosci. 1992;242:109-18.

\section{Correspondence}

Elisa Brietzke

Rua Pedro de Toledo, 669, $3^{\circ}$ andar, fundos, Vila Clementino

04039-032 - São Paulo, SP - Brazil

E-mail: elisabrietzke@hotmail.com 
Appendix 1

\section{Escala de Sintomas Prodrômicos de Transtorno Bipolar - BPSS}

SEÇÃO A 1: Informações Gerais: Data de Nascimento:

Etnia:

1. Diagnóstico Atual (marque todos que se aplicam):

1a. Episódio Atual (marque todos que se aplicam)

$\square$ Bipolar I $\square$ Bipolar II $\square$ Hipomania $\square$ Mania $\square$ Depressão $\square$ Misto

Bipolar SOE $\square$ Com sintomas psicóticos congruentes com o humor

$\square$ T. Humor SOE $\square$ Com sintomas psicóticos não congruentes com o humor

$\square$ Outro - Especificar .................... $\square$ em remissão parcial $\square$ em remissão completa

3. Gravidade dos Sintomas Atuais e Funcionamento do Paciente:

2a) Gravidade da Doença (Impressão Clínica Global-Gravidade):

1) $\square$ normal; 2) $\square$ limítrofe; 3) $\square$ leve; 4) $\square$ moderado; 5) $\square$ acentuada; 6) $\square$ grave 7) $\square$ extremo

2b) Avaliação Global do Funcionamento (AGF 0-100):

3. Quantas vezes você apresentou episódios ou períodos de humor alterado DESDE a primeira vez em que você ficou em mania ou em depressão até agora? Total \#:

SEÇÃO A 2 - Determinação das datas do Episódio de Humor e do Pródromo: Anote em papéis separados (Datas do episódio completo determinadas de acordo com a entrevista diagnóstica)

1. Quando você teve seu primeiro episódio depressivo [completo] (i.e., sentiu-se triste/"pra baixo", mais de 50\% do tempo e na maioria dos dias por 2 semanas ou mais em cada episódio, associado a $\geq 5 / 9$ critérios diagnósticos)? $\overline{\text { (mês) }} / \frac{}{\text { (ano) }}$ anos de idade (só anotar idade se não conseguir lembrar data)

2. Início do Pródromo: Quando você ou outra pessoa notaram os primeiros sintomas antes do episódio depressivo completo?

$$
\frac{}{\text { (mês) }} / \frac{}{\text { (ano) }} \text { anos de idade (só anotar idade se não conseguir lembrar data) }
$$

3. Quando você teve seu primeiro episódio de (hipo)mania/misto (i.e., por $\geq 4$ dias em cada episódio (i.e., TAB II se EDM presente) ou $\geq 7$ dias (ou se foi necessária internação/sintomas psicóticos) (i.e., TAB I), sentindo-se eufórico, no topo do mundo e/ou irritável $\geq 50 \%$ do tempo na maioria dos dias, associado a $\geq 3$ ( 4 se somente irritável)/7 critérios diagnósticos?

$$
\overline{\text { (mês) }}^{\prime} / \frac{}{\text { (ano) }} \text { anos de idade (só anotar idade se não conseguir lembrar data) }
$$

4. Início do Pródromo: Quando você ou outra pessoa notaram os primeiros sintomas antes do primeiro episódio de (hipo)mania / misto completo?

$$
\overline{\text { (mês) }}^{\prime} \frac{}{\text { (ano) }} \text { anos de idade (só anotar idade se não conseguir lembrar data) }
$$

SEÇÃO B - Descrição do PRÓDROMO do Primeiro Episódio de Depressão

"Eu vou perguntar sobre o período que antecedeu esse primeiro episódio de depressão"

1. Logo antes de você notar suas primeiras mudanças (logo antes do início do pródromo de depressão), qual era seu maior nível de funcionamento

Gravidade de Problemas Escolares:
$\square$ Ausente $\square$ Muito Leve $\square$ Leve $\square$
Gravidade de Problemas Sociais:
$\square$ Ausente $\square$ Muito Leve $\square$ Leve $\square$ Moderado $\square$ Acentuado $\square$ Grave $\square$ Extremo
Gravidade de Problemas Familiares:
$\square$ Ausente $\square$ Muito Leve $\square$ Leve $\square$ Moderado $\square$ Acentuado $\square$ Grave $\square$ Extremo
Gravidade Geral:
$\square$ Ausente $\square$ Muito Leve $\square$ Leve $\square$ Moderado $\square$ Acentuado $\square$ Grave $\square$ Extremo 
1a. Com relação à gravidade geral dos seus problemas, por quanto tempo você ficou se sentindo dessa maneira? (Tempo)

2. Na época do seu primeiro sintoma perceptível antes do primeiro episódio de depressão (início do pródromo de depressão), você vivenciou algum evento de vida traumático ou estressante?

Não $\quad$ Sim (ESPECIFICAR):

3. Padrão de Início: Durante o período entre os primeiros sintomas perceptíveis e o primeiro episódio completo de depressão, seus sintomas:

Iniciaram de gradualmente e pioraram lentamente $=1$

- Iniciaram de maneira relativamente gradual e pioraram rapidamente $=2$

_ Iniciaram subitamente - você estava bem num dia e doente no dia seguinte $=3$

4. Quem notou as primeiras mudanças (marque todos que se apliquem):

Criança [o proprio individuo?]

Escola

Colegas

Família

Pais de colegas

Profissional de saúde (Descreva):

Estranhos

5. Que tipo de tratamento você recebeu durante o período entre os primeiros sintomas perceptíveis e o primeiro episódio completo de depressão (marque todos que se apliquem)? Antidepressivos

Outro (descreva):

Antipsicóticos

Estabilizadores do humor Nenhum Estimulantes

Ansiolíticos

Psicoterapia

SEÇÃO B I - Perguntas Abertas sobre o Pródromo do Primeiro Episódio de Depressão :

No momento, quais você acha (ou o que outras pessoas lhe contaram) que foram seus primeiros sinais ou sintomas perceptíveis antes do primeiro episódio completo de depressão (ou seja, o pródromo)? Por favor, seja específico. (Você pode listar quantos sintomas desejar.)

! Entrevistador, certifique-se de repetir a data do início do pródromo e do início do primeiro episódio de depressão antes de preencher na tabela]

(*Entrevistador: anote primeiro o período, gravidade e frequência de todos os sintomas. Volte para avaliar relação com uso de substâncias APENAS se o paciente afirmou usar substâncias nesse período da sua vida (veja resposta ao à abaixo.)

\begin{tabular}{|c|c|c|c|c|c|c|c|c|c|c|c|c|c|c|c|c|c|}
\hline \multirow[t]{9}{*}{ Sintoma } & \multirow{2}{*}{$\begin{array}{l}\text { Início: \# } \\
\text { de meses } \\
\text { antes } \\
\text { do 10 } \\
\text { episódio } \\
\end{array}$} & \multicolumn{3}{|c|}{$\begin{array}{l}\text { Gravidade }{ }^{1} \\
\text { 1. Leve } \\
\text { 2. Moderado } \\
\text { 3. Grave }\end{array}$} & \multicolumn{4}{|c|}{$\begin{array}{l}\text { Frequência }{ }^{2} \\
\text { 1. Poucas vezes/raro } \\
\text { 2. Recorrente/ episódico } \\
\text { 3. Razoavelmente contínuo } \\
\text { 4. Por toda a vida/ } \\
\text { temperamento }\end{array}$} & \multicolumn{5}{|c|}{$\begin{array}{l}\text { Você usou drogas / álcool neste } \\
\text { período? } \\
\text { 1. > de } 1 \text { mês antes } \\
\text { 2. entre } 1 \text { mês e } 1 \text { dia antes } \\
\text { 3. no mesmo dia ou até } 1 \text { mês } \\
\text { depois } \\
\text { 4. > de } 1 \text { mês depois }\end{array}$} & \multicolumn{4}{|c|}{$\begin{array}{l}\text { Especificar o tipo } \\
\text { de substância } \\
\text { 1. álcool } \\
\text { 2. maconha } \\
\text { 3. cocaína } \\
\text { 4. outro (especificar) }\end{array}$} \\
\hline & & 1 & $\checkmark 2$ & $\checkmark 3$ & 1 & $\checkmark 2$ & 43 & $\checkmark 4$ & $\checkmark N$ & । 1 & $\checkmark 2$ & $\checkmark 3$ & $\checkmark 4$ & 1 & $\vdash 2$ & 13 & $\rightarrow 4$ \\
\hline & & $\checkmark 1$ & $\checkmark 2$ & 13 & 1 & $\checkmark 2$ & $\checkmark 3$ & $\checkmark 4$ & $N$ & $\checkmark 1$ & $\checkmark 2$ & $\checkmark 3$ & $\checkmark 4$ & 1 & $\checkmark 2$ & -3 & $\checkmark 4$ \\
\hline & & $\downarrow 1$ & $\vdash 2$ & $\vdash 3$ & 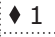 & $\vdash 2$ & $\bullet 3$ & $\bullet 4$ & $\checkmark N$ & $\bullet 1$ & $\checkmark 2$ & 13 & $\rightarrow 4$ & 1 & $\vdash 2$ & $\vdash 3$ & $\bullet 4$ \\
\hline & & $\downarrow 1$ & $\checkmark 2$ & $\checkmark 3$ & 1 & $\vdash 2$ & $\checkmark 3$ & $\checkmark 4$ & $N$ & $\checkmark 1$ & $\bullet 2$ & 13 & 4 & 1 & $\vdash 2$ & $\checkmark 3$ & $\rightarrow 4$ \\
\hline & & $\downarrow 1$ & $\vdash 2$ & $\vdash 3$ & $\downarrow 1$ & $\vdash 2$ & $\bullet 3$ & $\rightarrow 4$ & $\checkmark N$ & $\bullet 1$ & $\bullet 2$ & 13 & $\vdash 4$ & 11 & $\vdash 2$ & $\vdash 3$ & $\bullet 4$ \\
\hline & & 1 & $\checkmark 2$ & $\checkmark 3$ & 1 & $\checkmark 2$ & $\checkmark 3$ & - 4 & $-N$ & $\checkmark 1$ & $\checkmark 2$ & $\checkmark 3$ & $\checkmark 4$ & 1 & $\checkmark 2$ & $\checkmark 3$ & $\checkmark 4$ \\
\hline & & $\downarrow 1$ & $\vdash 2$ & $\vdash 3$ & 1 & $\vdash 2$ & $\vdash 3$ & $\bullet 4$ & 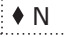 & $\downarrow 1$ & $\checkmark 2$ & 13 & -4 & 1 & $\downarrow 2$ & +3 & $\vdash 4$ \\
\hline & & 1 & $\checkmark 2$ & 13 & 1 & 12 & 13 & 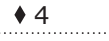 & $\sim \mathrm{N}$ & $\downarrow 1$ & $\checkmark 2$ & 13 & 4 & 1 & +2 & 13 & $\rightarrow 4$ \\
\hline
\end{tabular}

${ }^{1}$ Gravidade: 1 =leve: perceptível, não afeta o funcionamento; $2=$ moderado: claramente perceptível, pode afetar o funcionamento; 3 =grave: claramente perceptível, claramente afeta o funcionamento ${ }^{2}$ Frequência: 1 =infrequente: $<1 x /$ semana; $2=$ recorrente: $\geq 1 \times /$ semana, mas $<50 \%$; $3=$ continuo: $\geq 50 \%$; 4= por toda a vida: traço sem piora significativa (dupla codificação possível se o traço pré existente claramente piora durante o pródromo) 
$\diamond$ Você estava usando drogas ou álcool nesse período da sua vida? S / N

o Se sim, quais substâncias:

○ Quando você começou a usar cada uma delas?

o Com que frequência você usou cada uma delas?

O Que quantidade você usou dessas drogas?

o Se sim, você sente que algum dos sintomas acima poderia ter sido causado pelo uso de substâncias? S / N

O Quais sintomas?

(*Entrevistador: volte e marque as 2 últimas colunas para quaisquer sintomas que coincidiram com o uso de substâncias)

\section{SEÇ̃̃O B 2 - Perguntas Estruturadas sobre o Pródromo do 10 Episódio Completo de Depressão:}

"Você me contou alguns dos sintomas que você percebeu no período de tempo anterior ao seu primeiro episódio completo de depressão. Agora eu vou perguntar se você se lembra de ter certos sintomas específicos durante esse período".

! Entrevistador, certifique-se de repetir a data do início do pródromo e do início do primeiro episódio referido ao item!

Nota para o entrevistador: Marque "sim" apenas se os sintomas foram clinicamente relevantes. Use o seu julgamento. *Questione sobre a influência do uso de álcool / drogas apenas para os sintomas relatados, após a lista completa ter sido perguntada.

\begin{tabular}{|c|c|c|c|c|c|c|c|}
\hline $\begin{array}{l}\text { DURANTE O } \\
\text { PRÓDROMO DE } \\
\text { DEPRESSÃO }\end{array}$ & $\begin{array}{l}\text { Você teve } \\
\text { sintomas } \\
\text { perceptíveis } \\
\text { de: }\end{array}$ & $\begin{array}{l}\text { Início: } \\
\text { \# de } \\
\text { meses } \\
\text { antes } \\
\text { do 10 } \\
\text { episódio }\end{array}$ & $\begin{array}{l}\text { Gravidade }{ }^{1} \\
\text { 1. Leve } \\
\text { 2. Moderado } \\
\text { 3. Grave }\end{array}$ & $\begin{array}{l}\text { Frequência }{ }^{2} \\
\text { 1. Poucas vezes/ } \\
\text { infrequente } \\
\text { 2. Recorrente/episódico } \\
\text { 3. Razoavelmente contínuo } \\
\text { 4. Toda vida/ } \\
\text { temperamento }\end{array}$ & $\begin{array}{l}\text { Algum desses } \\
\text { foi parte do } \\
10 \text { episódio? }\end{array}$ & $\begin{array}{l}\text { Você usou drogas } \\
\text { / álcool neste } \\
\text { período? } \\
\text { 1. > de } 1 \text { mês } \\
\text { antes } \\
\text { 2. entre } 1 \text { mês e } 1 \\
\text { dia antes } \\
\text { 3. no mesmo dia ou } \\
\text { até } 1 \text { mês depois } \\
\text { 4. }>\text { de } 1 \text { mês } \\
\text { depois }\end{array}$ & $\begin{array}{l}\text { Indique } \\
\text { Substância }\end{array}$ \\
\hline $\begin{array}{l}\text { 1. Isolamento } \\
\text { social }\end{array}$ & $\checkmark S \bullet N$ & & $1 \cdot 2 \cdot 3$ & $\bullet \cdot 2 \cdot 3 \cdot 4$ & $\bullet S \bullet N$ & $\bullet N \cdot 1 \bullet 2 \cdot 3 \bullet 4$ & \\
\hline $\begin{array}{l}\text { 2. Queda do } \\
\text { funcionamento } \\
\text { na escola / } \\
\text { trabalho }\end{array}$ & $-S \cdot N$ & & $11 \cdot 2 \cdot 3$ & $\mid 1 \cdot 2 \cdot 3 \cdot 4$ & IS N & $N N \cdot 1 \cdot 2 \cdot 3 \cdot 4$ & \\
\hline $\begin{array}{l}\text { 3. Dificuldade } \\
\text { de se concentrar, } \\
\text { prestar atenção } \\
\text { ou se lembrar de } \\
\text { coisas }\end{array}$ & $\checkmark S \bullet N$ & & $11 \cdot 2 \cdot 3$ & $\bullet 1 \cdot 2 \cdot 3 \cdot 4$ & $-S \cdot N$ & $N N \cdot 1 \cdot 2 \cdot 3 \cdot 4$ & \\
\hline $\begin{array}{l}\text { 4. Dificuldade } \\
\text { em pensar ou } \\
\text { se comunicar } \\
\text { claramente? }\end{array}$ & o $\bullet N$ & & $11 \cdot 2 \cdot 3$ & $11 \cdot 2 \cdot 3 \cdot 4$ & $\checkmark S \cdot N$ & $\cdot N \cdot 1 \cdot 2 \cdot 3 \cdot 4$ & \\
\hline $\begin{array}{l}\text { 5. Ansiedade ou } \\
\text { nervosismo }\end{array}$ & $-S \bullet N$ & & $11 \cdot 2 \cdot 3$ & $\bullet 1 \cdot 2 \cdot 3 \cdot 4$ & $\| S \bullet N$ & $\mid N \cdot 1 \cdot 2 \cdot 3 \cdot 4$ & \\
\hline $\begin{array}{l}\text { 6. Obsessões e } \\
\text { compulsões }\end{array}$ & $\checkmark S \bullet N$ & & $11 \cdot 2 \cdot 3$ & $11 \cdot 2 \cdot 3 \cdot 4$ & $\bullet S \bullet N$ & $N \cdot 1 \cdot 2 \cdot 3 \cdot 4$ & \\
\hline $\begin{array}{l}\text { 7. Humor } \\
\text { depressivo }\end{array}$ & $\checkmark S \bullet N$ & & $11 \cdot 2 \cdot 3$ & $\bullet 1 \cdot 2 \cdot 3 \bullet 4$ & $\checkmark S \bullet N$ & $N N \cdot 1 \cdot 2 \cdot 3 \cdot 4$ & \\
\hline $\begin{array}{l}\text { 8. Irritabilidade } \\
\text { ou raiva }\end{array}$ & $-S \cdot N$ & & $11 \cdot 2 \cdot 3$ & $\cdot 1 \cdot 2 \cdot 3 \cdot 4$ & $-S \cdot N$ & $N N \cdot 1 \cdot 2 \cdot 3 \cdot 4$ & \\
\hline $\begin{array}{l}\text { 9. } \downarrow \text { no prazer / } \\
\text { interesse }\end{array}$ & $\checkmark S \bullet N$ & & $11 \cdot 2 \cdot 3$ & $\bullet 1 \cdot 2 \cdot 3 \cdot 4$ & $\sim S \bullet N$ & $\mid N \cdot 1 \cdot 2 \cdot 3 \cdot 4$ & \\
\hline $\begin{array}{l}\text { 10. Ganho de } \\
\text { peso ou } \uparrow \text { no } \\
\text { apetite }\end{array}$ & $-S \bullet N$ & & $11 \cdot 2 \cdot 3$ & $\bullet 1 \cdot 2 \cdot 3 \bullet 4$ & $\bullet S \bullet N$ & $n N \cdot 1 \cdot 2 \cdot 3 \cdot 4$ & \\
\hline
\end{tabular}


(cont.)

\begin{tabular}{|c|c|c|c|c|c|c|c|}
\hline $\begin{array}{l}\text { DURANTE O } \\
\text { PRÓDROMO DE } \\
\text { DEPRESSÃO }\end{array}$ & $\begin{array}{l}\text { Você teve } \\
\text { sintomas } \\
\text { perceptíveis } \\
\text { de: }\end{array}$ & \begin{tabular}{|l} 
Início: \\
\# de \\
meses \\
antes \\
do 10 \\
episódio
\end{tabular} & $\begin{array}{l}\text { Gravidade }{ }^{1} \\
\text { 1. Leve } \\
\text { 2. Moderado } \\
\text { 3. Grave }\end{array}$ & $\begin{array}{l}\text { Frequência } 2 \\
\text { 1. Poucas vezes/ } \\
\text { infrequente } \\
\text { 2. Recorrente/episódico } \\
\text { 3. Razoavelmente contínuo } \\
\text { 4. Toda vida/ } \\
\text { temperamento }\end{array}$ & $\begin{array}{l}\text { Algum desses } \\
\text { foi parte do } \\
1{ }^{\circ} \text { episódio? }\end{array}$ & $\begin{array}{l}\text { Você usou drogas } \\
\text { / álcool neste } \\
\text { período? } \\
1 .>\text { de } 1 \text { mês } \\
\text { antes } \\
2 . \text { entre } 1 \text { mês e } 1 \\
\text { dia antes } \\
\text { 3. no mesmo dia ou } \\
\text { até } 1 \text { mês depois } \\
4 .>\text { de } 1 \text { mês } \\
\text { depois }\end{array}$ & $\begin{array}{l}\text { Indique } \\
\text { Substância }\end{array}$ \\
\hline $\begin{array}{l}\text { 11. Perda de } \\
\text { peso ou } \downarrow \text { no } \\
\text { apetite }\end{array}$ & $\bullet S \bullet N$ & & $\bullet 1 \cdot 2 \cdot 3$ & $\bullet 1 \cdot 2 \cdot 3 \cdot 4$ & $\bullet S \bullet N$ & $\bullet N \cdot 1 \cdot 2 \cdot 3 \cdot 4$ & \\
\hline 12. Insônia & $\checkmark S \bullet N$ & & $\bullet 1 \bullet 2 \bullet 3$ & $\cdot 1 \cdot 2 \cdot 3 \cdot 4$ & $\checkmark S \bullet N$ & $\bullet N \cdot 1 \cdot 2 \bullet 3 \bullet 4$ & \\
\hline $\begin{array}{l}\text { 13. Dormindo } \\
\text { muito }\end{array}$ & $\bullet S \bullet N$ & & $\bullet 1 \cdot 2 \cdot 3$ & $\bullet 1 \cdot 2 \cdot 3 \cdot 4$ & $\bullet S \bullet N$ & $\bullet N \cdot 1 \bullet 2 \bullet 3 \bullet 4$ & \\
\hline $\begin{array}{l}\text { 14c. Troca do } \\
\text { dia pela noite } \\
\text { (Sono) }\end{array}$ & $\bullet S \cdot N$ & & $11 \cdot 2 \cdot 3$ & $1 \cdot 2 \cdot 3 \cdot 4$ & $\bullet S \cdot N$ & $\bullet N \cdot 1 \cdot 2 \cdot 3 \cdot 4$ & \\
\hline $\begin{array}{l}\text { 15. Fisicamente } \\
\text { lentificado }\end{array}$ & $\bullet S \bullet N$ & & $\cdot 1 \cdot 2 \cdot 3$ & $11 \cdot 2 \cdot 3 \cdot 4$ & $\bullet S \bullet N$ & $\bullet N \cdot 1 \cdot 2 \cdot 3 \cdot 4$ & \\
\hline $\begin{array}{l}\text { 16. Fisicamente } \\
\text { agitado }\end{array}$ & $\bullet S \cdot N$ & & $11 \cdot 2 \cdot 3$ & $\bullet 1 \cdot 2 \cdot 3 \cdot 4$ & $\checkmark S \bullet N$ & $\bullet N \cdot 1 \cdot 2 \cdot 3 \cdot 4$ & \\
\hline $\begin{array}{l}\text { 17. Cansaço ou } \\
\text { falta de energia }\end{array}$ & $\bullet S \cdot N$ & & - $1 \cdot 2 \cdot 3$ & $11 \cdot 2 \cdot 3 \cdot 4$ & $\bullet S \bullet N$ & $\bullet N \cdot 1 \cdot 2 \cdot 3 \cdot 4$ & \\
\hline $\begin{array}{l}\text { 18. Sentindo-se } \\
\text { sem valor ou } \\
\text { culpado (circule) }\end{array}$ & $\checkmark S \bullet N$ & & $11 \cdot 2 \cdot 3$ & $11 \cdot 2 \cdot 3 \cdot 4$ & $\bullet S \bullet N$ & $\bullet N \cdot 1 \cdot 2 \cdot 3 \cdot 4$ & \\
\hline $\begin{array}{l}\text { 19. Pensamentos } \\
\text { sobre suicídio }\end{array}$ & $\checkmark S \cdot N$ & & $11 \cdot 2 \cdot 3$ & $11 \cdot 2 \cdot 3 \cdot 4$ & $\checkmark S \cdot N$ & $\cdot N \cdot 1 \cdot 2 \cdot 3 \cdot 4$ & \\
\hline $\begin{array}{l}20 \text { Tentativas } \\
\text { de suicídio }\end{array}$ & $\bullet S \cdot N$ & & $11 \cdot 2 \cdot 3$ & $11 \cdot 2 \cdot 3 \cdot 4$ & $\checkmark S \bullet N$ & $\mapsto N \cdot 1 \cdot 2 \cdot 3 \cdot 4$ & \\
\hline $\begin{array}{l}\text { 21. Com- } \\
\text { portamento } \\
\text { auto-agressivo } \\
\text { (sem intenção } \\
\text { de se matar) } \\
\text { (Especifique): }\end{array}$ & $\checkmark S \cdot N$ & & $11 \cdot 2 \cdot 3$ & $11 \cdot 2 \cdot 3 \cdot 4$ & $\checkmark S \cdot N$ & $\cdot N \cdot 1 \cdot 2 \cdot 3 \cdot 4$ & \\
\hline $\begin{array}{l}\text { 22. Impaciente } \\
\text { ou com } \\
\text { dificuldade } \\
\text { para controlar } \\
\text { a raiva }\end{array}$ & $\bullet S \bullet N$ & & $11 \cdot 2 \cdot 3$ & $\bullet 1 \cdot 2 \cdot 3 \cdot 4$ & $\bullet S \cdot N$ & $N N \cdot 1 \cdot 2 \cdot 3 \cdot 4$ & \\
\hline $\begin{array}{l}\text { 23. Mudanças } \\
\text { frequentes, } \\
\text { labilidade de } \\
\text { humor }\end{array}$ & $\bullet S \cdot N$ & & $11 \cdot 2 \cdot 3$ & $11 \cdot 2 \cdot 3 \cdot 4$ & $\checkmark S \cdot N$ & $\mapsto N \cdot 1 \cdot 2 \cdot 3 \cdot 4$ & \\
\hline $\begin{array}{l}\text { 24. Excessiva- } \\
\text { mente alegre, } \\
\text { feliz, no topo } \\
\text { do mundo }\end{array}$ & $\checkmark S \bullet N$ & & $11 \cdot 2 \cdot 3$ & $11 \cdot 2 \cdot 3 \cdot 4$ & $\checkmark S \bullet N$ & N $1 \cdot 2 \cdot 3 \cdot 4$ & \\
\hline $\begin{array}{l}\text { 25. Falando } \\
\text { mais que o } \\
\text { normal }\end{array}$ & $\bullet S \bullet N$ & & $\cdot 1 \cdot 2 \cdot 3$ & $\cdot 1 \cdot 2 \cdot 3 \cdot 4$ & $\bullet S \bullet N$ & $\bullet N \cdot 1 \cdot 2 \cdot 3 \cdot 4$ & \\
\hline $\begin{array}{l}\text { 21. Pensamen- } \\
\text { to acelerado ou } \\
\text { pulando de um } \\
\text { pensamento a } \\
\text { outro }\end{array}$ & $\checkmark S \cdot N$ & & - $1 \cdot 2 \cdot 3$ & $11 \cdot 2 \cdot 3 \cdot 4$ & $\checkmark S \bullet N$ & $\bullet N \cdot 1 \cdot 2 \cdot 3 \cdot 4$ & \\
\hline $\begin{array}{l}\text { 26. Excesso de } \\
\text { energia }\end{array}$ & $\bullet S \bullet N$ & & $\cdot 1 \cdot 2 \cdot 3$ & $\bullet 1 \bullet 2 \bullet 3 \bullet 4$ & $\bullet S \bullet N$ & $\bullet N \cdot 1 \cdot 2 \cdot 3 \cdot 4$ & \\
\hline $\begin{array}{l}\text { 27. Desejo } \\
\text { sexual } \\
\text { aumentado }\end{array}$ & $\checkmark S \cdot N$ & & $11 \cdot 2 \cdot 3$ & $\cdot 1 \cdot 2 \cdot 3 \cdot 4$ & $\checkmark S \cdot N$ & $\cdot N \cdot 1 \cdot 2 \cdot 3 \cdot 4$ & \\
\hline $\begin{array}{l}28 \text { Comporta- } \\
\text { mento sexual } \\
\text { de risco }\end{array}$ & $\bullet S \bullet N$ & & $-1 \cdot 2 \cdot 3$ & $\cdot 1 \cdot 2 \cdot 3 \cdot 4$ & $\checkmark S \cdot N$ & N $11 \cdot 2 \cdot 3 \cdot 4$ & \\
\hline $\begin{array}{l}\text { 29. Diminuição } \\
\text { da necessidade } \\
\text { de sono }\end{array}$ & $\bullet S \bullet N$ & & $\cdot 1 \cdot 2 \cdot 3$ & $11 \cdot 2 \cdot 3 \cdot 4$ & $\bullet S \bullet N$ & $\bullet N \cdot 1 \cdot 2 \cdot 3 \cdot 4$ & \\
\hline
\end{tabular}




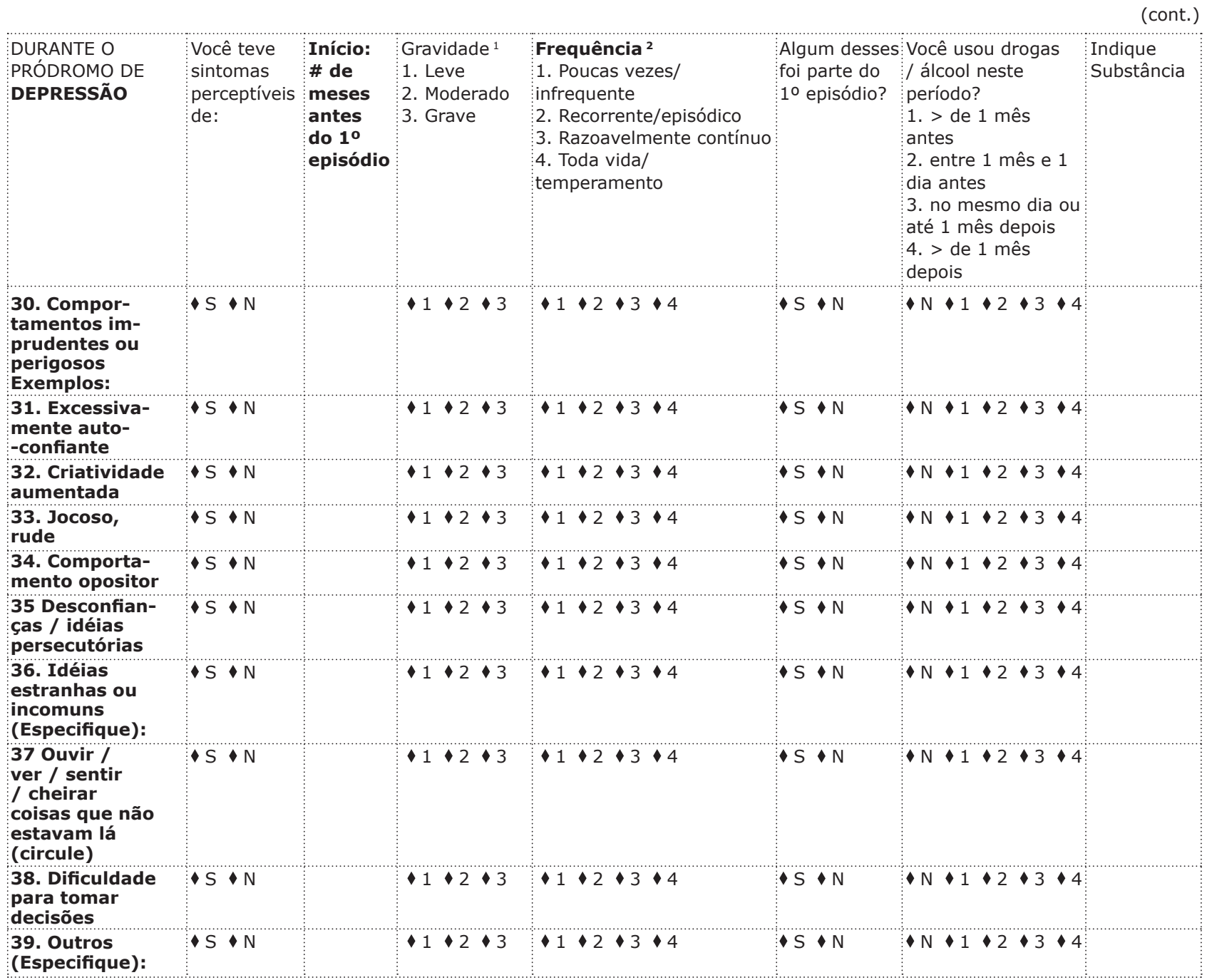

1 Gravidade: 1 =leve: perceptível, não afeta o funcionamento; $2=$ moderado: claramente perceptível, pode afetar o funcionamento; 3=grave: claramente perceptível, claramente afetando o funcionamento

${ }^{2}$ Erequência: 1 =infrequente: $<1 \times /$ semana; 2 =recorrenet: $\geq 1 \times /$ semana, mas $<50 \%$; $3=$ continuo: $\geq 50 \%$; $4=$ toda vida: traço sem piora significativa (dupla codificação possível se o traço pré existente claramente piora durante o pródromo)

\section{SEÇÃO C - Descrição do PRÓDROMO do 10 Episódio de Mania/Misto}

"Eu vou perguntar sobre o período que antecedeu esse primeiro episódio de mania / misto"

1. Logo antes de você notar suas primeiras mudanças (logo antes do início do pródromo de mania/misto),qual era seu maior nível de funcionamento

Gravidade de Problemas Escolares

$\square$ Ausente $\square$ Muito Leve $\square$ Leve $\square$ Moderado $\square$ Acentuado $\square$ Grave $\square$ Extremo

Gravidade de Problemas Sociais:

$\square$ Ausente $\square$ Muito Leve $\square$ Leve $\square$ Moderado $\square$ Acentuado $\square$ Grave $\square$ Extremo

Gravidade de Problemas Familiares:

$\square$ Ausente $\square$ Muito Leve $\square$ Leve $\square$ Moderado $\square$ Acentuado $\square$ Grave $\square$ Extremo

Gravidade Geral:

$\square$ Ausente $\square$ Muito Leve $\square$ Leve $\square$ Moderado $\square$ Acentuado $\square$ Grave $\square$ Extremo

1a. Com relação à gravidade geral dos seus problemas, por quanto tempo você ficou se sentindo dessa maneira? (Tempo) 
2. Na época do seu primeiro sintoma perceptível antes do primeiro episódio de mania/misto (início do pródromo de mania/misto), você vivenciou algum evento de vida traumático ou estressante? Não Sim (ESPECIFICAR):

3. Padrão de Início: Durante o período entre os primeiros sintomas perceptíveis e o primeiro episódio de mania/ misto, seus sintomas: Iniciaram de gradualmente e pioraram lentamente $=1$

_ Iniciaram de maneira relativamente gradual e pioraram rapidamente $=2$

_ Iniciaram subitamente - você estava bem num dia e doente no dia seguinte $=3$

4. Quem notou as primeiras mudanças (marque todos que se apliquem):

Criança [o proprio individuo?]
Estranhos $\_$Profissional de saúde (Descreva):

5. Que tipo de tratamento você recebeu durante o período entre os primeiros sintomas perceptíveis e o primeiro episódio de mania/misto (marque todos que se apliquem)?
Antidepressivos Estimulantes Outro (descreva):

_ Antipsicóticos Ansiolíticos Estabilizadores do humor Psicoterapia Nenhum

SEÇÃO C I - Perguntas Abertas sobre o Pródromo do Primeiro Episódio:

No momento, quais você acha (ou o que outras pessoas lhe contaram) que foram seus primeiros sinais ou sintomas perceptíveis antes do primeiro episódio de mania/misto (ou seja, o pródromo)? Por favor, seja específico. (Você pode listar quantos sintomas desejar.)

\section{! Entrevistador, certifique-se de repetir a data do início do pródromo e do início do primeiro episódio de mania/misto antes de preencher na tabela]}

(*Entrevistador: anote primeiro o período, gravidade e frequência de todos os sintomas. Volte para avaliar relação com uso de substâncias APENAS se o paciente afirmou usar substâncias nesse período da sua vida (veja resposta ao à abaixo.)

\begin{tabular}{|c|c|c|c|c|c|c|c|c|c|c|c|c|c|c|c|c|c|}
\hline \multirow[t]{10}{*}{ Sintoma } & \multirow{2}{*}{$\begin{array}{l}\text { Início: \# } \\
\text { de meses } \\
\text { antes } \\
\text { do } 10 \\
\text { episódio }\end{array}$} & \multicolumn{3}{|c|}{$\begin{array}{l}\text { Gravidade }{ }^{1} \\
\text { 1. Leve } \\
\text { 2. Moderado } \\
\text { 3. Grave }\end{array}$} & \multicolumn{4}{|c|}{$\begin{array}{l}\text { Frequência }{ }^{2} \\
\text { 1. Poucas vezes/raro } \\
\text { 2. Recorrente/ episódico } \\
\text { 3. Razoavelmente contínuo } \\
\text { 4. Por toda a vida/ } \\
\text { temperamento }\end{array}$} & \multicolumn{5}{|c|}{$\begin{array}{l}\text { Você usou drogas / álcool neste } \\
\text { período? } \\
\text { 1. > de } 1 \text { mês antes } \\
\text { 2. entre } 1 \text { mês e } 1 \text { dia antes } \\
\text { 3. no mesmo dia ou até } 1 \text { mês } \\
\text { depois } \\
\text { 4. > de } 1 \text { mês depois }\end{array}$} & \multicolumn{4}{|c|}{$\begin{array}{l}\text { Especificar o tipo } \\
\text { de substância } \\
\text { 1. álcool } \\
\text { 2. maconha } \\
\text { 3. cocaína } \\
\text { 4. outro (especificar) }\end{array}$} \\
\hline & & 1 & $\bullet 2$ & $\checkmark 3$ & 1 & $\checkmark 2$ & $\bullet 3$ & $\checkmark 4$ & $\diamond N$ & $\checkmark 1$ & $\bullet 2$ & $\checkmark 3$ & $\checkmark 4$ & $\checkmark 1$ & $\checkmark 2$ & $\checkmark 3$ & $\checkmark 4$ \\
\hline & & 1 & $\checkmark 2$ & $\checkmark 3$ & $\downarrow 1$ & $\checkmark 2$ & $\checkmark 3$ & $\checkmark 4$ & $\bullet N$ & $\downarrow 1$ & $\checkmark 2$ & $\checkmark 3$ & $\checkmark 4$ & 1 & $\checkmark 2$ & $\checkmark 3$ & $\bullet 4$ \\
\hline & & 1 & 12 & 13 & 1 & 12 & 13 & $\checkmark 4$ & $\bullet N$ & $\checkmark 1$ & 12 & 13 & - 4 & 1 & 12 & 13 & $\checkmark 4$ \\
\hline & & 1 & 12 & 13 & 1 & 12 & 13 & 4 & $1 N$ & 1 & 12 & 13 & 4 & 1 & 12 & 13 & $\checkmark 4$ \\
\hline & & 1 & 12 & 13 & 1 & 12 & 13 & 4 & $\checkmark N$ & $\checkmark 1$ & 12 & 13 & 4 & 1 & 12 & 13 & $\checkmark 4$ \\
\hline & & 1 & $\bullet 2$ & 13 & 1 & $\vdash 2$ & $\bullet 3$ & $\bullet 4$ & $\downarrow N$ & $\checkmark 1$ & $\bullet 2$ & $\bullet 3$ & $\checkmark 4$ & 1 & $\checkmark 2$ & $\bullet 3$ & $\checkmark 4$ \\
\hline & & 1 & $\checkmark 2$ & 13 & 1 & 12 & 13 & $\checkmark 4$ & $\bullet N$ & $\downarrow 1$ & 12 & 13 & - 4 & 1 & $\checkmark 2$ & 13 & $\checkmark 4$ \\
\hline & & 1 & 12 & 13 & 1 & 12 & 13 & 4 & $\checkmark N$ & 1 & 12 & 13 & - 4 & 1 & 12 & 13 & $\checkmark 4$ \\
\hline & & 1 & $\bullet 2$ & 13 & 1 & 12 & 13 & - 4 & $\checkmark N$ & 1 & 12 & 13 & $\checkmark 4$ & 1 & 12 & 13 & $\checkmark 4$ \\
\hline
\end{tabular}

Gravidade: 1 =leve: perceptível, não afeta o funcionamento; $2=$ moderado: claramente perceptível, pode afetar o funcionamento; 3 =grave: claramente perceptível, claramente afeta o funcionamento ${ }^{2}$ Frequência: 1 =infrequente: < 1x/semana; $2=$ recorrente: $\geq 1 \mathrm{x} /$ semana, mas $<50 \%$; $3=$ continuo: $\geq 50 \%$; $4=$ por toda a vida: traço sem piora significativa (dupla codificação possível se o traço pré existente claramente piora durante o pródromo)

$\diamond$ Você estava usando drogas ou álcool nesse período da sua vida? S / N o Se sim, quais substâncias:

O Quando você começou a usar cada uma delas?

o Com que frequência você usou cada uma delas?

○ Que quantidade você usou dessas drogas? 
O Quais sintomas?

(*Entrevistador: volte e marqueas 2 últimas colunas para quaisquer sintomas que coincidiram com o uso de substâncias) SECÃ̃O C 2 - Perguntas Estruturadas sobre o Pródromo do 10 Episódio Completo de Mania/Misto:

"Você me contou alguns dos sintomas que você percebeu no período de tempo anterior ao seu primeiro episódio de mania/misto. Agora eu vou perguntar se você se lembra de ter certos sintomas específicos durante esse período". ! Entrevistador, certifique-se de repetir a data do início do pródromo e do início do primeiro episódio referido ao item!

Nota para o entrevistador: Marque "sim" apenas se os sintomas foram clinicamente relevantes. Use o seu julgamento. *Questione sobre a influência do uso de álcool / drogas apenas para os sintomas relatados, após a lista completa ter sido perguntada.

\begin{tabular}{|c|c|c|c|c|c|c|c|}
\hline $\begin{array}{l}\text { DURANTE O } \\
\text { PRÓDROMO DE } \\
\text { MANIA/MISTO }\end{array}$ & $\begin{array}{l}\text { Você teve } \\
\text { sintomas } \\
\text { perceptíveis } \\
\text { de: }\end{array}$ & $\begin{array}{l}\text { Início: } \\
\text { \# de } \\
\text { meses } \\
\text { antes } \\
\text { do 10 } \\
\text { episódio }\end{array}$ & $\begin{array}{l}\text { Gravidade }{ }^{1} \\
\text { 1. Leve } \\
\text { 2. Moderado } \\
\text { 3. Grave }\end{array}$ & $\begin{array}{l}\text { Frequência }{ }^{2} \\
\text { 1. Poucas vezes/ } \\
\text { infrequente } \\
\text { 2. Recorrente/episódico } \\
\text { 3. Razoavelmente contínuo } \\
\text { 4. Toda vida/ } \\
\text { temperamento }\end{array}$ & $\begin{array}{l}\text { Algum desses } \\
\text { foi parte do } \\
10 \text { episódio? }\end{array}$ & $\begin{array}{l}\text { Você usou drogas } \\
\text { / álcool neste } \\
\text { período? } \\
1 .>\text { de } 1 \text { mês } \\
\text { antes } \\
\text { 2. entre } 1 \text { mês e } 1 \\
\text { dia antes } \\
\text { 3. no mesmo dia ou } \\
\text { até } 1 \text { mês depois } \\
4 .>\text { de } 1 \text { mês } \\
\text { depois }\end{array}$ & $\begin{array}{l}\text { Indique } \\
\text { Substância }\end{array}$ \\
\hline $\begin{array}{l}\text { 1. Isolamento } \\
\text { social }\end{array}$ & $\checkmark \mathrm{S} \bullet \mathrm{N}$ & & $\bullet 1 \cdot 2 \cdot 3$ & $\cdot 1 \cdot 2 \cdot 3 \cdot 4$ & $\bullet S \bullet N$ & $N N \cdot 1 \cdot 2 \cdot 3 \cdot 4$ & \\
\hline $\begin{array}{l}\text { 2. Queda do } \\
\text { funcionamento } \\
\text { na escola / } \\
\text { trabalho }\end{array}$ & $-S \cdot N$ & & $11 \cdot 2 \cdot 3$ & $11 \cdot 2 \cdot 3 \cdot 4$ & $\checkmark S \cdot N$ & $N \cdot 1 \cdot 2 \cdot 3 \cdot 4$ & \\
\hline $\begin{array}{l}\text { 3. Dificuldade } \\
\text { de se concentrar, } \\
\text { prestar atenção } \\
\text { ou se lembrar de } \\
\text { coisas }\end{array}$ & $\checkmark s \cdot N$ & & $1 \cdot 2 \cdot 3$ & $1 \cdot 2 \cdot 3 \cdot 4$ & $\checkmark s \cdot N$ & $N \cdot 1 \cdot 2 \cdot 3 \cdot 4$ & \\
\hline $\begin{array}{l}\text { 4. Dificuldade } \\
\text { em pensar ou } \\
\text { se comunicar } \\
\text { claramente? }\end{array}$ & $\checkmark S \cdot N$ & & $11 \cdot 2 \cdot 3$ & $1 \cdot 2 \cdot 3 \cdot 4$ & $-S \cdot N$ & $n \cdot 1 \cdot 2 \cdot 3 \cdot 4$ & \\
\hline $\begin{array}{l}\text { 5. Ansiedade ou } \\
\text { nervosismo }\end{array}$ & $\checkmark S \bullet N$ & & $11 \cdot 2 \cdot 3$ & $\cdot 1 \cdot 2 \cdot 3 \cdot 4$ & $\bullet S \bullet N$ & $N \cdot 1 \cdot 2 \cdot 3 \cdot 4$ & \\
\hline $\begin{array}{l}\text { 6. Obsessões e } \\
\text { compulsões }\end{array}$ & $\bullet S \cdot N$ & & $11 \cdot 2 \cdot 3$ & $1 \cdot 2 \cdot 3 \cdot 4$ & $-S \cdot N$ & $N \cdot 1 \cdot 2 \cdot 3 \cdot 4$ & \\
\hline $\begin{array}{l}\text { 7. Humor } \\
\text { depressivo }\end{array}$ & $\bullet S \bullet N$ & & $11 \cdot 2 \cdot 3$ & $1 \cdot 2 \cdot 3 \cdot 4$ & $\bullet S \cdot N$ & $N \cdot 1 \cdot 2 \cdot 3 \cdot 4$ & \\
\hline $\begin{array}{l}\text { 8. Irritabilidade } \\
\text { ou raiva }\end{array}$ & $\bullet S \bullet N$ & & $11 \cdot 2 \cdot 3$ & $1 \cdot 2 \cdot 3 \cdot 4$ & $\bullet S \bullet N$ & $N \cdot 1 \cdot 2 \cdot 3 \cdot 4$ & \\
\hline $\begin{array}{l}\text { 9. } \downarrow \text { no prazer / } \\
\text { interesse }\end{array}$ & $-S \cdot N$ & & $11 \cdot 2 \cdot 3$ & $1 \cdot 2 \cdot 3 \cdot 4$ & $\checkmark S \bullet N$ & $N \cdot 1 \cdot 2 \cdot 3 \cdot 4$ & \\
\hline $\begin{array}{l}\text { 10. Ganho de } \\
\text { peso ou } \uparrow \text { no } \\
\text { apetite }\end{array}$ & $\bullet S \bullet N$ & & $11 \cdot 2 \cdot 3$ & $\bullet 1 \cdot 2 \cdot 3 \cdot 4$ & $-S \bullet N$ & $N \cdot 1 \cdot 2 \cdot 3 \cdot 4$ & \\
\hline $\begin{array}{l}\text { 11. Perda de } \\
\text { peso ou } \downarrow \text { no } \\
\text { apetite }\end{array}$ & $-S \cdot N$ & & $11 \cdot 2 \cdot 3$ & $11 \cdot 2 \cdot 3 \cdot 4$ & $-S \cdot N$ & $N \cdot 1 \cdot 2 \cdot 3 \cdot 4$ & \\
\hline 12. Insônia & $\checkmark S \bullet N$ & & $\cdot 1 \cdot 2 \cdot 3$ & $11 \cdot 2 \cdot 3 \cdot 4$ & $\checkmark S \bullet N$ & $n+1 \cdot 2 \cdot 3 \cdot 4$ & \\
\hline $\begin{array}{l}\text { 13. Dormindo } \\
\text { muito }\end{array}$ & $\checkmark S \cdot N$ & & $11 \cdot 2 \cdot 3$ & $1 \cdot 2 \cdot 3 \cdot 4$ & $\checkmark S \cdot N$ & $N \cdot 1 \cdot 2 \cdot 3 \cdot 4$ & \\
\hline $\begin{array}{l}\text { 14c. Troca do } \\
\text { dia pela noite } \\
\text { (Sono) }\end{array}$ & $\checkmark S \cdot N$ & & $11 \cdot 2 \cdot 3$ & $1 \cdot 2 \cdot 3 \cdot 4$ & $\checkmark S \bullet N$ & $N \cdot 1 \cdot 2 \cdot 3 \cdot 4$ & \\
\hline $\begin{array}{l}\text { 15. Fisicamente } \\
\text { lentificado }\end{array}$ & $\bullet S \bullet N$ & & $11 \cdot 2 \cdot 3$ & $1 \cdot 2 \cdot 3 \cdot 4$ & $\bullet S \bullet N$ & $\mid N \cdot 1 \cdot 2 \cdot 3 \cdot 4$ & \\
\hline $\begin{array}{l}\text { 16. Fisicamente } \\
\text { agitado }\end{array}$ & $\bullet S \bullet N$ & & $11 \cdot 2 \cdot 3$ & $1 \cdot 2 \cdot 3 \cdot 4$ & $\checkmark S \bullet N$ & $N \cdot 1 \cdot 2 \cdot 3 \cdot 4$ & \\
\hline
\end{tabular}


(cont.)

\begin{tabular}{|c|c|c|c|c|c|c|c|}
\hline $\begin{array}{l}\text { DURANTE O } \\
\text { PRÓDROMO DE } \\
\text { MANIA/MISTO }\end{array}$ & $\begin{array}{l}\text { Você teve } \\
\text { sintomas } \\
\text { perceptíveis } \\
\text { de: }\end{array}$ & $\begin{array}{l}\text { Início: } \\
\text { \# de } \\
\text { meses } \\
\text { antes } \\
\text { do 10 } \\
\text { episódio }\end{array}$ & $\begin{array}{l}\text { Gravidade }{ }^{1} \\
\text { 1. Leve } \\
\text { 2. Moderado } \\
\text { 3. Grave }\end{array}$ & $\begin{array}{l}\text { Frequência }{ }^{2} \\
\text { 1. Poucas vezes/ } \\
\text { infrequente } \\
\text { 2. Recorrente/episódico } \\
\text { 3. Razoavelmente contínuo } \\
\text { 4. Toda vida/ } \\
\text { temperamento }\end{array}$ & $\begin{array}{l}\text { Algum desses } \\
\text { foi parte do } \\
10 \text { episódio? }\end{array}$ & $\begin{array}{l}\text { Você usou drogas } \\
\text { / álcool neste } \\
\text { período? } \\
1 .>\text { de } 1 \text { mês } \\
\text { antes } \\
\text { 2. entre } 1 \text { mês e } 1 \\
\text { dia antes } \\
\text { 3. no mesmo dia ou } \\
\text { até } 1 \text { mês depois } \\
4 .>\text { de } 1 \text { mês } \\
\text { depois }\end{array}$ & $\begin{array}{l}\text { Indique } \\
\text { Substância }\end{array}$ \\
\hline $\begin{array}{l}\text { 17. Cansaço ou } \\
\text { falta de energia }\end{array}$ & $\bullet S \bullet N$ & & $11 \cdot 2 \cdot 3$ & $11 \cdot 2 \cdot 3 \cdot 4$ & $\checkmark S \bullet N$ & N $11 \cdot 2 \cdot 3 \cdot 4$ & \\
\hline $\begin{array}{l}\text { 18. Sentindo-se } \\
\text { sem valor ou } \\
\text { culpado (circule) }\end{array}$ & $\checkmark S \cdot N$ & & $11 \cdot 2 \cdot 3$ & $11 \cdot 2 \cdot 3 \cdot 4$ & $\bullet S \cdot N$ & * $11 \cdot 2 \cdot 3 \cdot 4$ & \\
\hline $\begin{array}{l}\text { 19. Pensamentos } \\
\text { sobre suicídio }\end{array}$ & $\checkmark S \cdot N$ & & $1 \cdot 2 \cdot 3$ & $11 \cdot 2 \cdot 3 \cdot 4$ & - S N N & N $11 \cdot 2 \cdot 3 \cdot 4$ & \\
\hline $\begin{array}{l}20 \text { Tentativas } \\
\text { de suicídio }\end{array}$ & $\checkmark S \cdot N$ & & $11 \cdot 2 \cdot 3$ & $11 \cdot 2 \cdot 3 \cdot 4$ & $-S \cdot N$ & $\mid N \cdot 1 \cdot 2 \cdot 3 \cdot 4$ & \\
\hline $\begin{array}{l}\text { 21. Com- } \\
\text { portamento } \\
\text { auto-agressivo } \\
\text { (sem intenção } \\
\text { de se matar) } \\
\text { (Especifique): }\end{array}$ & $\checkmark S \cdot N$ & & $\cdot 1 \cdot 2 \cdot 3$ & $11 \cdot 2 \cdot 3 \cdot 4$ & $\bullet S \bullet N$ & N $11 \cdot 2 \cdot 3 \cdot 4$ & \\
\hline $\begin{array}{l}\text { 22. Impaciente } \\
\text { ou com } \\
\text { dificuldade } \\
\text { para controlar } \\
\text { a raiva }\end{array}$ & $\checkmark S \bullet N$ & & $11 \cdot 2 \cdot 3$ & $11 \cdot 2 \cdot 3 \cdot 4$ & $\bullet S \bullet N$ & N $11 \cdot 2 \cdot 3 \cdot 4$ & \\
\hline $\begin{array}{l}\text { 23. Mudanças } \\
\text { frequentes, } \\
\text { labilidade de } \\
\text { humor }\end{array}$ & $\checkmark S \bullet N$ & & $11 \cdot 2 \cdot 3$ & $11 \cdot 2 \cdot 3 \cdot 4$ & $\bullet S \bullet N$ & $\mid N \cdot 1 \cdot 2 \cdot 3 \cdot 4$ & \\
\hline $\begin{array}{l}\text { 24. Excessiva- } \\
\text { mente alegre, } \\
\text { feliz, no topo } \\
\text { do mundo }\end{array}$ & $\bullet S \bullet N$ & & $11 \cdot 2 \cdot 3$ & $11 \cdot 2 \cdot 3 \cdot 4$ & $\checkmark S \bullet N$ & $\bullet N \cdot 1 \cdot 2 \cdot 3 \cdot 4$ & \\
\hline $\begin{array}{l}\text { 25. Falando } \\
\text { mais que o } \\
\text { normal }\end{array}$ & $\checkmark S \bullet N$ & & $11 \cdot 2 \cdot 3$ & $1 \cdot 2 \cdot 3 \cdot 4$ & $\checkmark S \bullet N$ & N $11 \cdot 2 \cdot 3 \cdot 4$ & \\
\hline $\begin{array}{l}\text { 21. Pensamen- } \\
\text { to acelerado ou } \\
\text { pulando de um } \\
\text { pensamento a } \\
\text { outro }\end{array}$ & $\bullet S \bullet N$ & & $11 \cdot 2 \cdot 3$ & $11 \cdot 2 \cdot 3 \cdot 4$ & $\bullet S \bullet N$ & $\mid N \cdot 1 \cdot 2 \cdot 3 \cdot 4$ & \\
\hline $\begin{array}{l}\text { 26. Excesso de } \\
\text { energia }\end{array}$ & $\checkmark S \bullet N$ & & $11 \cdot 2 \cdot 3$ & $11 \cdot 2 \cdot 3 \cdot 4$ & $\checkmark S \bullet N$ & $N \cdot 1 \cdot 2 \cdot 3 \cdot 4$ & \\
\hline $\begin{array}{l}\text { 27. Desejo } \\
\text { sexual } \\
\text { aumentado }\end{array}$ & $\checkmark S \bullet N$ & & $\cdot 1 \cdot 2 \cdot 3$ & $11 \cdot 2 \cdot 3 \cdot 4$ & $\checkmark S \bullet N$ & $\bullet N \cdot 1 \cdot 2 \cdot 3 \cdot 4$ & \\
\hline $\begin{array}{l}28 \text { Comporta- } \\
\text { mento sexual } \\
\text { de risco }\end{array}$ & $\checkmark S \bullet N$ & & $11 \cdot 2 \cdot 3$ & $11 \cdot 2 \cdot 3 \cdot 4$ & $-S \cdot N$ & $\mid N \cdot 1 \cdot 2 \cdot 3 \cdot 4$ & \\
\hline $\begin{array}{l}\text { 29. Diminuição } \\
\text { da necessidade } \\
\text { de sono }\end{array}$ & $\checkmark S \cdot N$ & & $11 \cdot 2 \cdot 3$ & $11 \cdot 2 \cdot 3 \cdot 4$ & $\bullet S \cdot N$ & $n+1 \cdot 2 \cdot 3 \cdot 4$ & \\
\hline $\begin{array}{l}\text { 30. Compor- } \\
\text { tamentos im- } \\
\text { prudentes ou } \\
\text { perigosos } \\
\text { Exemplos: }\end{array}$ & $\checkmark S \bullet N$ & & $\bullet 1 \cdot 2 \cdot 3$ & $\bullet 1 \cdot 2 \cdot 3 \cdot 4$ & $\bullet S \bullet N$ & $\bullet N \cdot 1 \cdot 2 \cdot 3 \cdot 4$ & \\
\hline $\begin{array}{l}\text { 31. Excessiva- } \\
\text { mente auto- } \\
\text { confiante }\end{array}$ & $\checkmark S \bullet N$ & & $11 \cdot 2 \cdot 3$ & $11 \cdot 2 \cdot 3 \cdot 4$ & $\bullet S \bullet N$ & $\mid N \cdot 1 \cdot 2 \cdot 3 \cdot 4$ & \\
\hline $\begin{array}{l}\text { 32. Criatividade } \\
\text { aumentada }\end{array}$ & $\checkmark S \bullet N$ & & $\cdot 1 \cdot 2 \cdot 3$ & $\cdot 1 \cdot 2 \cdot 3 \cdot 4$ & $\bullet S \bullet N$ & $\bullet N \cdot 1 \cdot 2 \cdot 3 \cdot 4$ & \\
\hline $\begin{array}{l}\text { 33. Jocoso, } \\
\text { rude }\end{array}$ & $\bullet S \cdot N$ & & $11 \cdot 2 \cdot 3$ & $1 \cdot 2 \cdot 3 \cdot 4$ & $\checkmark S \bullet N$ & $\cdot N \cdot 1 \cdot 2 \cdot 3 \cdot 4$ & \\
\hline $\begin{array}{l}\text { 34. Comporta- } \\
\text { mento opositor }\end{array}$ & $\sim S \bullet N$ & & $1 \cdot 2 \cdot 3$ & $11 \cdot 2 \cdot 3 \cdot 4$ & - S N N & $+N \cdot 1 \cdot 2 \cdot 3 \cdot 4$ & \\
\hline
\end{tabular}

(cont.) 


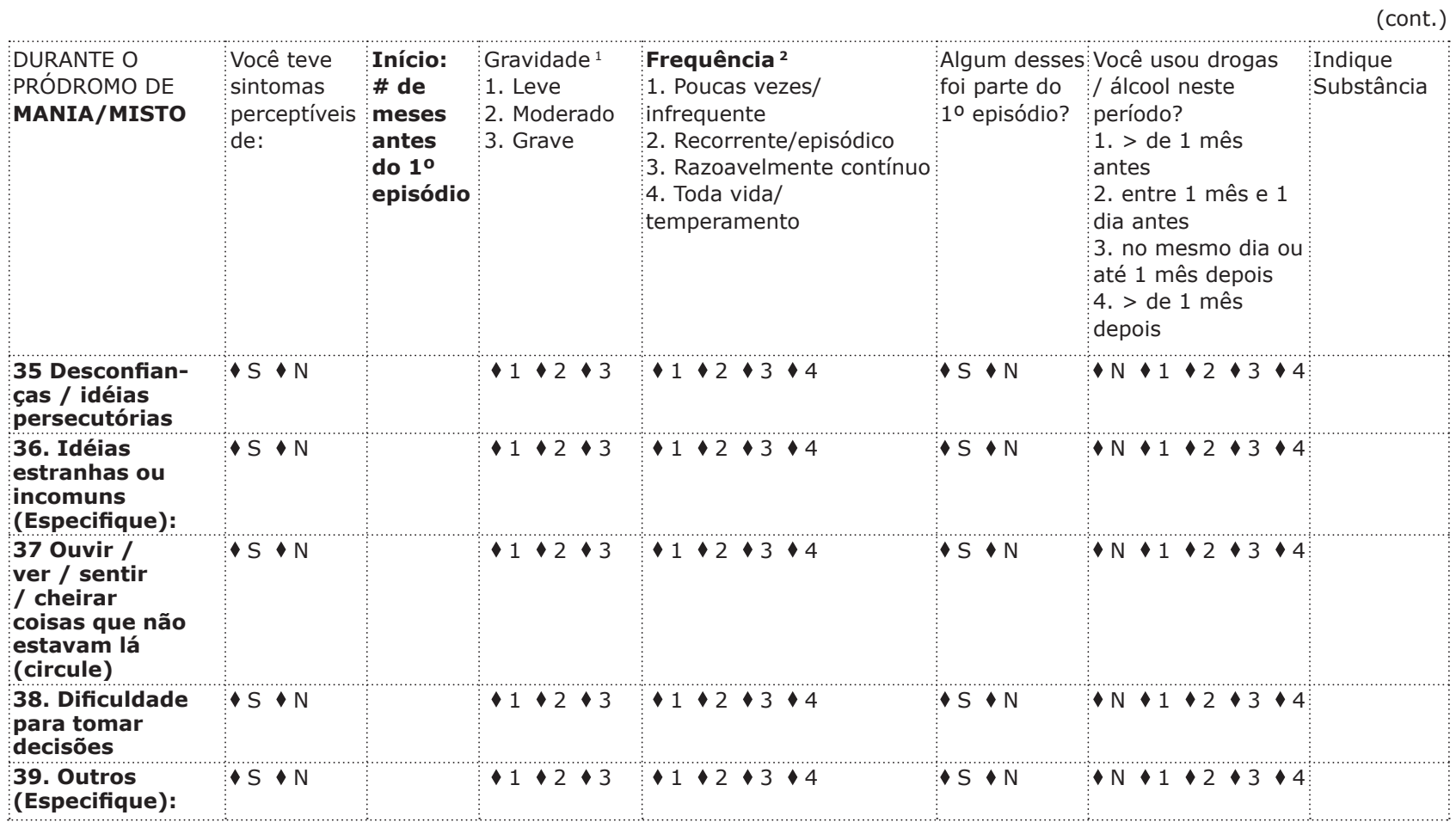

1ravidade: 1 =leve: perceptível, não afeta o funcionamento; $2=$ moderado: claramente perceptível, pode afetar o funcionamento; 3 =grave: claramente perceptível, claramente afetando o funcionamento

${ }^{2}$ Frequência: 1 =infrequente: $<1 \mathrm{x} /$ semana; $2=$ recorrenet: $\geq 1 \mathrm{x} /$ semana, mas $<50 \%$; $3=$ continuo: $\geq 50 \%$; 4=toda vida: traço sem piora significativa (dupla codificação possível se o traço pré existente claramente piora durante o pródromo)

SEÇÃO D - Informação Adicional sobre Episódios Sindromicos de Humor: Eventos de Vida, Tempo de Diagnóstico, Hospitalizacões e Suicídio

\section{D.1 DEPRESSÃO}

1. Na época do seu primeiro episódio depressivo completo de depressão, você vivenciou algum evento de vida traumático ou estressante? Não . . . . . . . . . . . . . . Sim (ESPECIFICAR):

2. Quando você foi diagnosticado pela primeira vez com depressão? (mês) $/ \overline{\text { ano })}$ anos de idade (só anotar idade se não conseguir lembrar data)

3. Quando foi sua primeira hospitalização psiquiátrica por um episódio de depressão? $\overline{\text { (mês) }} / \overline{\text { ano) }}$ anos de idade (só anotar idade se não conseguir lembrar data)

4. Quantas hospitalizações você já teve devido a episódios de depressão? .

\section{D.2 EPISÓDIO DE MANIA/MISTO}

1. Na época do seu primeiro episódio de mania / misto completo, você vivenciou algum evento de vida traumático ou estressante? Não $\quad$ Sim (ESPECIFICAR):

2. Quando você foi diagnosticado pela primeira vez com transtorno bipolar?

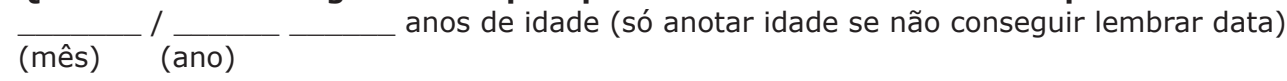


3. Quando foi sua primeira hospitalização psiquiátrica devido a um episódio de mania / misto (circule aquele que se aplica)? $\overline{\text { (mês) }}{ }^{\prime}(\overline{\text { ano })} \longrightarrow$ anos de idade (só anotar idade se não conseguir lembrar data)

4. Quantas hospitalizações você já teve devido a episódios de mania / misto?

\section{D.3 Hospitalizações associadas a comportamento suicida}

1. Quantas hospitalizações você teve devido a pensamentos/atitudes suicidas? \# durante episódio de depressão: ; \# durante episódio de mania/misto:

\# nem em mania ou depressão

2. Quantas hospitalizações você teve devido a tentativas de suicídio? \# durante episódio de depressão: ; \# durante episódio de mania/misto: \# nem em mania ou depressão

\section{D.4 - DURAÇ̃̃O DA CICLAGEM E FUNCIONAMENTO INTEREPISÓDICO}

1. Quantos episódios da primeira polaridade, p.ex., polaridade oposta, p.ex., ?: você teve antes do primeiro episódio da

2. Durante o período entres os primeiros sintomas perceptíveis do primeiro episódio de humor e o primeiro episódio da polaridade oposta, por favor especifique a duração mínima e máximo, assim como o tempo total passado em cada um dos seguintes estados de humor

\begin{tabular}{|c|c|c|c|c|}
\hline Episódio Afetivo & Duração Mínima & Duração Máxima & Duração "Típica" & Percentual Total de Tempo \\
\hline \multicolumn{5}{|l|}{ Depressão } \\
\hline \multicolumn{5}{|l|}{ Mania } \\
\hline \multicolumn{5}{|l|}{ Irritável } \\
\hline \multicolumn{5}{|l|}{ Misto } \\
\hline Eutimia & & & & \\
\hline
\end{tabular}

SEÇÃO E - Nível de Certeza quanto às Informações Recordadas:

Qual é nível de certeza das informações que você forneceu: Por cento. 